\title{
A Novel Null Space-Based Kernel Discriminant Analysis for Face Recognition
}

\author{
Tuo Zhao ${ }^{1}$, Zhizheng Liang ${ }^{1}$, David Zhang ${ }^{2}$, and Yahui Liu ${ }^{1}$ \\ ${ }^{1}$ Harbin Institute of Technology \\ tourzhao@gmail.com \\ ${ }^{2}$ Hongkong Polytechnic University
}

\begin{abstract}
The symmetrical decomposition is a powerful method to extract features for image recognition. It reveals the significant discriminative information from the mirror image of symmetrical objects. In this paper, a novel null space kernel discriminant method based on the symmetrical method with a weighted fusion strategy is proposed for face recognition. It can effectively enhance the recognition performance and shares the advantages of Null-space, kernel and symmetrical methods. The experiment results on ORL database and FERET database demonstrate that the proposed method is effective and outperforms some existing subspace methods.
\end{abstract}

Keywords: symmetrical decomposition, symmetrical null-space based kernel LDA, weighted fusion strategy, face recognition.

\section{Introduction}

Linear Discriminant Analysis (LDA) is a popular method in extracting features, and it has been successfully applied to many fields. In general, the objective of LDA is to seek a linear projection from the image space to a low dimensional space by maximizing the between-class scatter and minimizing the within-class scatter simultaneously. It is shown that LDA is better than PCA especially under illumination variation [1]. In [2], Zhao also favorably supported this point under the FERET testing framework. However, in many practical applications of LDA, when the number of the samples is much smaller than the dimension of the sample space, it results in the small size sample (SSS) problem [3]. During the past several decades, many methods have been proposed to deal with this problem. Belhumeur [1] used PCA to reduce the dimension of the samples to an intermediate dimension and then Fisher LDA is used to extract the features. However, the Fisher LDA only uses the regularized space of the within-class scatter matrix. Considering this point, $\mathrm{Yu}$ [4] proposed Direct LDA. In their method, they projected all the samples directly by removing the null space of between-class scatter matrix. Chen [5] made a more crucial modification. That is, they firstly projected all the samples onto the null space of within-class scatter matrix before doing DLDA and proposed a more powerful Null-space Based LDA (NLDA).

Although the methods mentioned above have proved to be efficient for face recognition, they are still linear techniques in nature. Hence they are inadequate to 
describe the complexity of real face images because of illumination, facial expression and pose variations. To deal with this problem, kernel methods including Kernel PCA (KPCA) and Kernel LDA [6, 7] are proposed. The idea of kernel methods is to map all the data by a non-linear function into the feature space and then to perform some operations in this space.

In recent years, some researchers introduced the symmetric idea in face recognition. Yang [8] presented a symmetrical principal component analysis (SPCA) algorithm according to the symmetry of human faces. Their method can reduce the sensitivities to outliers. In this paper, we propose a symmetrical null space-based kernel LDA method, which can simultaneously provide the advantages of kernel, symmetrical and Null-space methods.

The rest of this paper is organized as follows. Section 2 reviews the related work on Kernel Fisher and its variants. Section 3 proposes our method. The experimental results are shown in section 4 . Section 5 gives discussion and conclusions.

\section{Related Work}

\subsection{Fundamentals}

In Kernel methods, the input space $R^{n}$ firstly is mapped into the feature space $F$ by a non-linear mapping $\phi$, denoted as:

$$
\phi: R^{n} \rightarrow F, x \mapsto \phi(x)
$$

Kernel Fisher Discriminant is to solve the problem of LDA in the feature space $F$ by a set of nonlinear discriminant vectors in input space. By maximizing the following Fisher criterion:

$$
J^{\phi}(\varphi)=\frac{\left|\varphi^{T} S_{b}^{\phi} \varphi\right|}{\left|\varphi^{T} S_{t}^{\phi} \varphi\right|}
$$

where $S_{b}^{\phi}$ and $S_{t}^{\phi}$ are defined as below:

$$
\begin{gathered}
S_{b}^{\phi}=\frac{1}{M} \sum_{i=1}^{c} l_{i}\left(m_{i}^{\phi}-m^{\phi}\right)\left(m_{i}^{\phi}-m^{\phi}\right)^{T}, \\
S_{t}^{\phi}=\frac{1}{M} \sum_{i=1}^{M}\left(\phi\left(x_{i}\right)-m^{\phi}\right)\left(\phi\left(x_{i}\right)-m^{\phi}\right)^{T},
\end{gathered}
$$

where $x_{1}, x_{2}, \ldots, x_{M}$ is a set of $M$ training samples in input space, $l_{i}$ is the number of training samples of class $i$ and satisfies $\sum_{i=1}^{c} l_{i}=M, m_{i}^{\phi}$ is the mean vector of the mapped training samples of class $i$, and $m^{\phi}$ is the mean vector across all mapped training samples. 


\subsection{Kernel Fisher and Its Variant (KFD and NKLDA)}

Actually, we can obtain the discriminant vectors with the respect to the Fisher criterion by computing the Eigenvalue problem as $S_{b}^{\phi} \varphi=\lambda S_{t}^{\phi} \varphi$. In theory, any solution $\varphi \in F$ must lie in the span of all samples in $F$, so we can view $\varphi$ as a linear combination of $\phi\left(x_{i}\right)$ :

$$
\varphi=\sum_{j=1}^{M} a_{j} \phi\left(x_{j}\right)=Q \alpha,
$$

where $Q=\left[\phi\left(x_{1}\right), \phi\left(x_{2}\right), \ldots, \phi\left(x_{M}\right)\right]$ and $\alpha=\left(a_{1}, a_{2}, \ldots, a_{M}\right)^{T}$.

The matrix $K$ is defined as

$$
K=\tilde{K}-1_{M} \tilde{K}-\tilde{K} 1_{M}+1_{M} \tilde{K} 1_{M} .
$$

Here $1_{M}=(1 / M)_{M \times M}, \tilde{K}=Q^{T} Q$ is an $M \times M$ matrix and its elements are

$$
\tilde{K}_{i j}=\phi\left(x_{i}\right)^{T} \phi\left(x_{j}\right)=\left(\phi\left(x_{i}\right) \cdot \phi\left(x_{j}\right)\right)=k\left(x_{i}, y_{j}\right)
$$

corresponding to a given nonlinear mapping $\phi$ and, the new sample vector by kernel tricks, $K\left(x_{i}\right)$, is defined as a column of $K$ :

$$
K\left(x_{i}\right)=\left[k\left(x_{1}, x_{i}\right), k\left(x_{2}, x_{i}\right), \ldots, k\left(x_{M}, x_{i}\right)\right]^{T} .
$$

Substituting Eq.(5) into Eq.(2) gives

$$
J^{K}(\alpha)=\frac{\left|\alpha^{T} K_{b} \alpha\right|}{\left|\alpha^{T} K_{t} \alpha\right|},
$$

where the new between-class scatter matrix $K_{b}$ and total scatter matrix $K_{t}$ are

$$
\begin{gathered}
K_{b}=\frac{1}{M} \sum_{i=1}^{c} l_{i}\left(m_{i}-m\right)\left(m_{i}-m\right)^{T}, \\
K_{t}=\frac{1}{M} \sum_{i=1}^{M}\left(K\left(x_{i}\right)-m\right)\left(K\left(x_{i}\right)-m\right)^{T},
\end{gathered}
$$

where $m_{i}$ is the mean vector of all $K\left(x_{j}\right)$ belonging to class $i$, and $m$ is the mean vector of all $K\left(x_{j}\right)$. Eq.(9) is a standard Fisher equation and we obtain the final discriminant vectors by:

$$
X_{i}=\alpha \cdot K\left(x_{i}\right)
$$

Liu [8] developed kernel null space method. The between class scatter matrix $K_{b}$ is projected onto the null space of new within-class scatter matrix $K_{w}$. That is, the null space of $K_{w}$ in the mapping space is firstly calculated as 


$$
Y^{T} K_{w} Y=0,
$$

where $Y$ consists of the eigenvectors with zero eigenvalues and $Y^{T} Y=I$. Then we obtain

$$
\tilde{K}_{b}=Y^{T} K_{b} Y .
$$

Furthermore, the eigenvectors $U$ of $\tilde{K}_{b}$ with the first several largest eigenvalues are selected to form the transformation matrix

$$
X_{i}=U^{T} Y^{T} K\left(x_{i}\right) .
$$

\section{Symmetrical Null-Space Based Kernel LDA (SNKLDA)}

\subsection{Symmetrical Ideas}

For any function $f$, it can be written as $f=f_{e}+f_{o}$, in which $f_{e}=\left(f+f_{m}\right) / 2$ and $f_{o}=\left(f-f_{m}\right) / 2$. Here $f_{m}$ is the symmetrical counterpart of $f \cdot f_{e}$ and $f_{o}$ are, respectively, described by a linear combination of a set of even or odd symmetrical basis functions. Thus, any function can be linearly reconstructed by the two basis functions, one even symmetrical and another odd symmetrical. This is the even-odd decomposition principle. We can apply this principle to face images and define symmetry to be the horizontal mirror symmetry with the vertical midline of the image as its axis. According to the odd-even decomposition theory, $x_{i}$ can be decomposed as $x_{i}=x_{e i}+x_{o i}$ with $x_{e i}=\left(x_{i}+x_{m i}\right) / 2$ denoting the odd symmetrical image, and $x_{e i}=\left(x_{i}-x_{m i}\right) / 2$ denoting the odd symmetrical image, where $x_{m i}$ is the mirror image of $x_{i}$. Based on this, symmetrical methods perform classification on two new symmetrical image sets, and then combine the features of odd and even images as the final features.

\subsection{Our Proposed Method}

Theorem: For any polynomial kernel function $\phi$ and any two vectors $x_{a}$ and $x_{b}$, if $\left\langle x_{a}, x_{b}\right\rangle=0$, there exits a relationship: $\phi\left(x_{a}+x_{b}\right)=\phi\left(x_{a}\right)+\phi\left(x_{b}\right)$

Proof: Because $\phi(x)$ is a polynomial function, $\phi(x)$ can be written as $\phi(x)=\sum_{i=0}^{N} a_{i} x^{i}$, and similarly $\phi\left(x_{a}+x_{b}\right)=\sum_{i=0}^{N} a_{i}\left(x_{a}+x_{b}\right)^{i}$. Since $<x_{a}, x_{b}>=0$,

$$
\begin{aligned}
\phi\left(x_{a}+x_{b}\right)= & \sum_{i=0}^{N} a_{i}\left(x_{a}+x_{b}\right)^{i} \\
& =\sum_{i=0}^{N} \beta_{i}^{0} a_{i} x_{a}^{i}+\ldots+\sum_{i=0}^{N} \beta_{i}^{j} a_{i} x_{a}^{j} x_{b}^{i-j}+\ldots+\sum_{i=0}^{N} \beta_{i}^{i} a_{i} x_{b}^{i}
\end{aligned}
$$




$$
\begin{aligned}
& =\sum_{i=0}^{N} a_{i} x_{a}^{i}+\sum_{i=0}^{N} a_{i} x_{b}^{i} \\
& =\phi\left(x_{a}\right)+\phi\left(x_{b}\right)
\end{aligned}
$$

where $\beta$ is the coefficients of a linear combination.

So for new training samples in the feature space, the following equation holds

$$
\begin{aligned}
K_{i j} & =<\phi\left(x_{i}\right), \phi\left(x_{j}\right)> \\
& =<\phi\left(x_{o i}+x_{e i}\right), \phi\left(x_{o j}+x_{e j}\right)> \\
& =<\phi\left(x_{o i}\right)+\phi\left(x_{e i}\right), \phi\left(x_{o i}\right)+\phi\left(x_{e j}\right)> \\
& =<\phi\left(x_{o i}\right), \phi\left(x_{o j}\right)>+<\phi\left(x_{o i}\right), \phi\left(x_{e j}\right)>+<\phi\left(x_{e i}\right), \phi\left(x_{o j}\right)>+<\phi\left(x_{e i}\right), \phi\left(x_{e j}\right)>.
\end{aligned}
$$

Since the polynomial kernel $K_{i j}=<\phi\left(x_{i}\right), \phi\left(x_{j}\right)>=\left(<x_{i}, x_{j}>+1\right)^{d}$ is adopted for the symmetrical decomposition. Then we obtain:

$$
\begin{aligned}
& <\phi\left(x_{o i}\right), \phi\left(x_{e j}\right)>=\left(<x_{o i}, x_{e j}>+1\right)^{d}=(0+1)^{d}=1, \\
& <\phi\left(x_{e i}\right), \phi\left(x_{o j}\right)>=\left(<x_{e i}, x_{o j}>+1\right)^{d}=(0+1)^{d}=1 .
\end{aligned}
$$

Thus, it means that $k\left(x_{o i}, x_{e j}\right)$ and $k\left(x_{e i}, x_{o j}\right)$ do not contain the valid discriminant information. In [10], Lu et al. discussed the orthogonally of Kernel Symmetrical PCA in the original space and used the polynomial kernel in their experiments. But we find that in the feature space the inner product of $\phi\left(x_{o}\right)$ and $\phi\left(x_{e}\right)$ are constant when the polynomial kernel is applied. Therefore, when a symmetrical method adopts the polynomial kernel, it still only considers the principal components of even and odd symmetrical images, not considering the correlative components between even and odd symmetrical images. Therefore, when the Kernel Symmetrical PCA method adopts the polynomial kernel, we can decompose the sample set into two new sets. Then we can perform classification on even and odd images by combing their features.

Since NKLDA is more effective than KPCA in solving small size sample problem [6]. In the following, we combine it with symmetrical ideas in discriminant analysis to improve the classification performance. To this end, we propose SNKLDA. The proposed method utilizes the symmetrical properties to do NKLDA in both the even and odd space and it shares the advantages of two methods in some sense.

In the following, we describe the proposed method.

We firstly convert the training samples into two training sets $\left\{x_{e i}\right\}_{1 \leq i \leq M}$ and $\left\{x_{o i}\right\}_{1 \leq i \leq M}$. Then we project the new sample set in the feature space by the kernel trick. That is, we can obtain the following new kernel training sets:

$$
\begin{aligned}
& K_{e}\left(x_{e i}\right)=\left(k\left(x_{e 1}, x_{e i}\right), k\left(x_{e 2}, x_{e i}\right), \ldots, k\left(x_{e M}, x_{e i}\right)\right)^{T}, 1 \leq i \leq M, \\
& K_{o}\left(x_{o i}\right)=\left(k\left(x_{o 1}, x_{o i}\right), k\left(x_{o 2}, x_{o i}\right), \ldots, k\left(x_{o M}, x_{o i}\right)\right)^{T}, 1 \leq i \leq M .
\end{aligned}
$$

Then we calculate class mean, total mean, the between-class scatter matrix and the within-class scatter matrix of each new kernel training set as follows: 


$$
\begin{gathered}
m_{e j}=\sum_{j \in c} K\left(x_{e j}\right) / M, m_{e}=\sum_{j \in c} m_{e j} / c, m_{o j}=\sum_{j \in c} K\left(x_{o j}\right) / M, m_{o}=\sum_{j \in c} m_{o_{j}} / c \\
K_{w e}=\sum_{j=1}^{c} \sum_{i \in C_{j}}\left(K\left(x_{e i}\right)-m_{e j}\right)\left(K\left(x_{e i}\right)-m_{e j}\right)^{T} \\
K_{w o}=\sum_{j=1}^{c} \sum_{i \in C_{j}}\left(K\left(x_{o i}\right)-m_{o j}\right)\left(K\left(x_{o i}\right)-m_{o j}\right)^{T}, \\
K_{b e}=\sum_{i \in C_{j}}\left(m_{e j}-m_{e}\right)\left(m_{e j}-m_{e}\right)^{T}, K_{b o}=\sum_{i \in C_{j}}\left(m_{o j}-m_{o}\right)\left(m_{o j}-m_{o}\right)^{T}
\end{gathered}
$$

Based on the above steps, we perform feature extraction in both the odd and even spaces. That is, we extract the null space $Y_{e}$ of $K_{w e}$ and $Y_{o}$ of $K_{w o}$ such that

$$
Y_{e}^{T} K_{w e} Y_{e}=0, Y_{o}^{T} K_{w o} Y_{o}=0 .
$$

Subsequently, the between class scatter matrix $K_{b e}$ and $K_{b o}$ are projected onto the null space of $K_{w e}$ and $K_{w o}$

$$
\tilde{K}_{b e}=Y_{e}^{T} K_{b e} Y_{e}, \tilde{K}_{b o}=Y_{o}^{T} K_{b o} Y_{o} .
$$

Furthermore, the eigenvectors $U_{e}$ of $\tilde{K}_{b e}$ and $U_{o}$ of $\tilde{K}_{b o}$ with the first several largest eigenvalues are selected to form the transformation matrix

$$
X_{e}=U_{e}^{T} Y_{e}^{T} K_{e}\left(x_{e}\right), X_{o}=U_{o}^{T} Y_{o}^{T} K_{o}\left(x_{o}\right) .
$$

It is obvious that we get two sets of features from Eq. (24). It is necessary to use these features for classification. Note that in the previous symmetrical methods, the features are usually directly combined as the final features $[9,10]$. Different from the previous methods, we assign different weights to fuse the features in the odd and even spaces to improve the classification performance. The final features are defined as

$$
\begin{aligned}
& X_{\text {fuse }}(x)=\left[w_{e} X_{e}(x), w_{o} X_{o}(x)\right], \\
& w_{o}=\left(\frac{R_{o}}{R_{e}+R_{o}}\right)^{t}, w_{e}=\left(\frac{R_{e}}{R_{e}+R_{o}}\right)^{t}
\end{aligned}
$$

where $w_{o}$ and $w_{e}$ are fused weights, $R_{e}$ and $R_{o}$ are the correct recognition rates with even features and odd respectively, and $t=0,1,2$ in our experiment. To reduce the computational complexity of weight training, we compute the weights on a small set of samples.

\section{Experimental Results}

In order to compare the performance of SKNLDA with other methods, the experiments are performed on two popular face databases: ORL database and FERET database. All the following experiments share the same steps. The image data are 
preprocessed including histogram equalization, normalization. For the sake of simplicity, we choose the Nearest Neighbor as the classifier and the polynomial kernel function is selected as $k(x, y)=(1+\langle x, y\rangle)^{d}$ with degree $d=2$. In addition, we select $c-1$ and $N-1$ as the finial dimensions in Fisher LDA and KSPCA.

\subsection{Experiments on ORL Database}

There are 10 different images for each subject in ORL face database composed of 40 distinct subjects. All the subjects are in up-right, frontal position. The size of each face image is downsampled to $46 \times 56$. Fig. 1 shows all 10 images of the first person after histogram equalization.

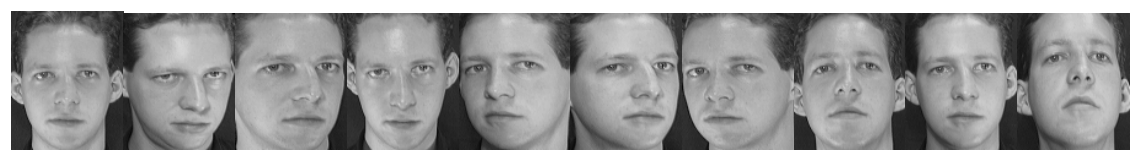

Fig. 1. Samples from the first person in ORL Database

In this set of experiments, the first 10 subjects in the database are selected to determine the weights: In order to reduce variation, we random select 2 images from each subject as training samples and the others are testing samples. The results are the average of 20 runs. We obtain even CRR 96.44\% and odd CRR: 59.00\%. Table 1 shows the performance of SNKLDA under different $t$.

Table 1. The performance of SNKLDA under different $t$ on ORL database

\begin{tabular}{llll}
\hline $\boldsymbol{K}$ & $t=0$ & $t=1$ & $t=2$ \\
\hline $\mathbf{2}$ & 85.39 & 86.44 & 86.33 \\
$\mathbf{3}$ & 91.35 & 92.03 & 91.88 \\
$\mathbf{4}$ & 94.52 & 94.82 & 94.75 \\
\hline
\end{tabular}

Table 2. The experiment results on ORL database

\begin{tabular}{llllll}
\hline $\boldsymbol{K}$ & Fisher & NLDA & NKLDA & KSPCA & SNKLDA \\
\hline $\mathbf{2}$ & 75.72 & 85.26 & 84.13 & 84.22 & 86.44 \\
$\mathbf{3}$ & 86.31 & 91.06 & 91.02 & 89.78 & 92.03 \\
$\mathbf{4}$ & 91.40 & 94.18 & 94.42 & 92.99 & 94.82 \\
\hline
\end{tabular}

Then we compare SNKLDA with some methods in the case of different numbers of training samples. The number of training samples per subject, $k$, varies from 2 to 4 . In each round, $k$ images are randomly selected from the database for training and the remaining images of the same subject are used for testing. For each $k, 50$ tests are performed and the final results are averaged over 50 runs. Table 2 shows the CRR $(\%)$ and we can see that the performance of SNKLDA is better than other methods. 


\subsection{Experiments on FERET Database}

In order to further test the capability of the proposed method, experiments are made on the dataset with more subjects such as the FERET database. In the experiments, we select a subset including 200 subjects respectively from FERET database with 11 upright, frontal-view images of each subject. The face images on this database involve much more variations in lighting, view and expressions. The size of face images is downsampled to $50 \times 60$. Fig. 2 shows all 11 images of the first person after histogram equalization.

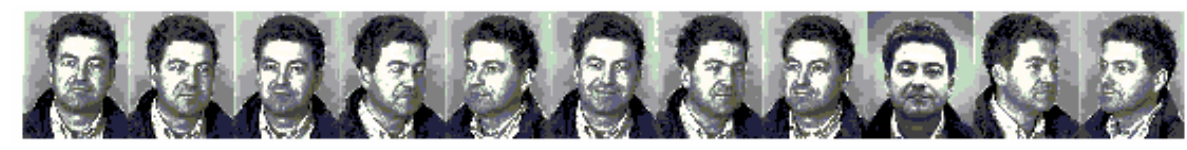

Fig. 2. Samples from the first person in FERET Database after histogram equalization

In this set of experiments, the first 40 subjects in the database are selected to determine the weights. In order to reduce variation, we random select 2 images from each subject as training samples and the others are testing samples. The results are the average of 20 runs. We obtain even CRR $80.81 \%$ and odd CRR: $20.56 \%$. Table 3 shows the performance of SNKLDA under different $t$.

Table 3. The performance of SNKLDA under different $t$ on ORL database

\begin{tabular}{llll}
\hline $\boldsymbol{K}$ & $t=0$ & $t=1$ & $t=2$ \\
\hline $\mathbf{2}$ & 66.70 & 68.07 & 67.99 \\
$\mathbf{3}$ & 73.21 & 74.67 & 74.64 \\
$\mathbf{4}$ & 77.42 & 78.34 & 78.27 \\
\hline
\end{tabular}

Table 4. The experiment results on ORL database

\begin{tabular}{llllll}
\hline $\boldsymbol{K}$ & Fisher & NLDA & NKLDA & KSPCA & SNKLDA \\
\hline $\mathbf{2}$ & 31.20 & 60.00 & 62.25 & 56.94 & 68.07 \\
$\mathbf{3}$ & 55.64 & 64.60 & 68.47 & 63.88 & 74.67 \\
$\mathbf{4}$ & 68.16 & 68.81 & 74.00 & 67.93 & 78.34 \\
\hline
\end{tabular}

The number of samples in each class, $k$, varies from 2 to 4 . For each $k, 100$ tests are performed and the final results are averaged over 100 runs. Table 4 shows the experimental results and we can see SNKLDA outperforms all the other subspace methods in all the cases.

\section{Discussions and Conclusions}

From the above two experiments we can find that SNKLDA is better than all the other methods. It may come from the fact that the SNKLDA method has the advantages of 

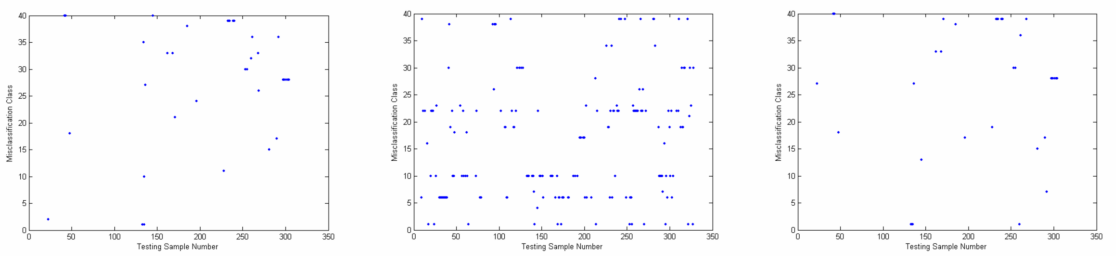

Fig. 3. Misclassification in even (L), odd (M) and full space with weighted strategy(R) on ORL

the NKLDA method in the small sample size problem. Although SNKLDA has twice computational complexity of NKLDA due to the symmetrical decomposition, the symmetry decomposition contributes to about $5 \%$ increases on FERET. In the above experiments the number of the subspace dimension is $(c-1) \times 2$ which is much lower than PCA method in the symmetrical methods.

It should be noted that extracting complementary discriminative information and the fusion strategy are also important for symmetrical methods. From the experiment we can see our weighted strategy is better than directly combination. In addition, from Fig. 3, we note that the two spaces are not complementary enough to increase the recognition rate greatly by the basic fusion strategy. The misclassified samples in the even space are usually also misclassified in the odd space. Hence how to efficiently use the complementary information in two spaces deserves the further research.

In all, the main contributions of this paper are briefly summarized as follows: (a) Proposed SNKLDA method that can provide better performance than other methods; (b) Proposed weighted strategy for even and odd features and revealed the bottleneck of the symmetrical method;(d) pointed out that the SKPCA method in the polynomial kernel still only considers the principal components of even and odd symmetrical images, not considering the correlative components between even and odd symmetrical images. However, considering the problems in the above discussion, there still has some wok to do in the near future. That is, we will pay much more attention to the following two aspects: (1) Fusion strategy: a more powerful fusion strategy should be used to maximize the utilization of the complementary information; (2) Kernel function: a novel kernel function should be used to avoid the orthogonality of even and odd features to generate a mixed space for classification.

\section{References}

1. Belhumeur, P.N., Hespanha, J.P., Kiregman, D.J.: Eigenfaces vs. Fisherfaces: Recognition Using Class Specific Linear Projection. IEEE Trans. on PAMI. 19(7), 711-720 (1997)

2. Zhao, W., Chellappa, R., Philips, P.J.: Subspace Linear Discriminant Analysis for Face Recognition. Tech Report CAR-TR-914 Center for Automation Research, University of Maryland (1999)

3. Fukunaga, K.: Introduction to Statistical Pattern Recognition. Academic Press, London (1990)

4. Yu, H., Yang, J.: A Direct LDA algorithm for High-dimensional Data with Application to Face Recognition. Pattern Recognition 34(10), 2067-2070 (2001) 
5. Chen, L.F., Liao, H.Y.M., Lin, J.C., Ko, M.T., Yu, G.J.: A new LDA-based Face Recognition System Which Can Solve the Small Sample Size Problem. Pattern Recognition 33(10), 1713-1726 (2000)

6. Yang, M.H.: Kernel Eigenfaces vs. Kernel Fisherfaces: Face Recognition Using Kernel Methods. In: Int. Conf. on Automatic Face and Gesture Recognition, pp. 215-220 (2002)

7. Liu, W., Wang, Y.H., Li, S.Z., Tan, T.N.: Null Space-based Kernel Fisher Discriminant Analysis for Face Recognition. In: Int. Conf. on Automatic Face and Gesture Recognition, pp. 369-374 (2004)

8. Yang, Q., Ding, X.: Symmetrical PCA in Face Recognition. In: IEEE Int. Conf. on Image Processing, pp. 97-100 (2002)

9. Lu, C., Zhang, C., Zhang, T., Zhang, W.: Kernel Based Symmetrical Principal Component Analysis for Face Classification. Nerucomputing 70(4-6), 904-911 (2007)

\section{Appendix}

Let a sample vector be $x=\left\{x_{1}, x_{2}, \ldots, x_{n-1}, x_{n}\right\}$ and its mirror vector be $x_{m}=\left\{x_{n}, x_{n-1}, \ldots, x_{2}, x_{1}\right\}$, and another sample vector be $y=\left\{y_{1}, y_{2}, \ldots, y_{n-1}, y_{n}\right\}$ and its mirror vector be $y_{m}=\left\{y_{n}, y_{n-1}, \ldots, y_{2}, y_{1}\right\}$. Then by symmetrical decomposition, we obtain

$$
\begin{aligned}
& x_{e}=\frac{x+x_{m}}{2}=\left\{\frac{x_{1}+x_{n}}{2}, \frac{x_{2}+x_{n-1}}{2}, \ldots, \frac{x_{n-1}+x_{2}}{2}, \frac{x_{n}+x_{1}}{2}\right\}, \\
& x_{o}=\frac{x-x_{m}}{2}=\left\{\frac{x_{1}-x_{n}}{2}, \frac{x_{2}-x_{n-1}}{2}, \ldots, \frac{x_{n-1}-x_{2}}{2}, \frac{x_{n}-x_{1}}{2}\right\}, \\
& y_{e}=\frac{y+y_{m}}{2}=\left\{\frac{y_{1}+y_{n}}{2}, \frac{y_{2}+y_{n-1}}{2}, \ldots, \frac{y_{n-1}+y_{2}}{2}, \frac{y_{n}+y_{1}}{2}\right\}, \\
& y_{o}=\frac{y-y_{m}}{2}=\left\{\frac{y_{1}-y_{n}}{2}, \frac{y_{2}-y_{n-1}}{2}, \ldots, \frac{y_{n-1}-y_{2}}{2}, \frac{y_{n}-y_{1}}{2}\right\} .
\end{aligned}
$$

The inner product is

$$
<x_{e}, y_{o}>=\left(\left(\frac{x_{1}+y_{n}}{2}\right)\left(\frac{x_{1}-y_{n}}{2}\right)+\ldots+\left(\frac{x_{n}+y_{1}}{2}\right)\left(\frac{x_{n}-y_{1}}{2}\right)\right) .
$$

It is obvious that $\left(\frac{x_{i}+y_{n-i}}{2}\right)\left(\frac{x_{i}-y_{n-i}}{2}\right)+\left(\frac{x_{n-i}+y_{i}}{2}\right)\left(\frac{x_{n-i}-y_{i}}{2}\right)=\left(\frac{x_{i}+y_{n-i}}{2}\right)\left(\frac{x_{i}-y_{n-i}}{2}\right)+$ $\left(-\left(\frac{x_{n-i}+y_{i}}{2}\right)\left(\frac{x_{i}-y_{n-i}}{2}\right)\right)=0$, so

$$
<x_{e}, y_{o}>=0 .
$$

In the similar way, we prove

$$
<x_{o}, y_{e}>=0 .
$$

